\title{
Requirements Identification for Real-Time Anomaly Detection in Industrie 4.0 Machine Groups: A Structured Literature Review
}

\author{
Philip Stahmann \\ University of Osnabrueck \\ philip.stahmann@uni-osnabrueck.de
}

\author{
Prof. Dr.-Ing. Bodo Rieger \\ University of Osnabrueck \\ bodo.rieger@uni-osnabrueck.de
}

\begin{abstract}
Industrie 4.0 environments generate an unprecedented amount of production data. This is due to the rising number of sensors and interconnected devices capable of emitting data in millisecond frequencies. Streaming analytics offers promising methodologies that can support handling and analysis of data volume and variety. Transparency and control over real-time data can increase production efficiency in tightly connected machine environments. Data transparency may avoid time-consuming assessment of machines to detect anomalous machine behavior causing production inefficiencies or failures. This paper aims to identify requirements to implement streaming analytics for the detection of anomalies in Industrie 4.0 production machine groups through a structured literature review.
\end{abstract}

\section{Introduction}

The digitalization radically changes how manufacturing companies operate. Technological advancements, such as the embedding of sensor technology, allow for closer integration of machines and production data. The virtual representation of physical production activities through sensor data supposedly enables enhancement of production planning and control [1]. Recent digital advancements in manufacturing have spawned the fourth industrial revolution, denominated "Industrie 4.0" in Germany. In Industrie 4.0, interdependent machines cooperate and communicate with each other [2]. The integration of machines to groups supposedly increases manufacturing flexibility and shall cater to the needs of individualized production. On the downside of machine integration, unexpected behavior in one machine may lead to failure in a connected machine. The assessment of the entire group to find the failure's root is time-consuming, especially when the assessment bases on stored, time-lagged data. Causal relationships in machine groups lead to a greater variety in potential failures in machine groups as compared to single machines [3]. Expert judgement as recently applied method to detect unexpected behavior is time lagged and potentially subjective [4]. Also, small deviations from expected machine group behavior that do not result in failure, but lead to inefficiencies might remain unnoticed by experts. Analyzing production data in real-time seems to be a promising approach to overcome the outlined disadvantages. In contrast to batch data analysis and expert assessment, real-time analysis requires data to arrive in a continuous stream, which imposes specific challenges. The term "streaming analytics" subsumes methodologies to approach these challenges [5]. The immediate identification of unexpected behavior may constitute a basis for real-time decision making to mitigate failures. To understand what is needed for real-time anomaly detection, this paper addresses the following research question:

What are the requirements to implement real-time anomaly detection in machine groups in Industrie 4.0?

The paper structures into six chapters. Chapter two goes into detail on the structured literature review used as methodology to identify requirements. Chapter three briefly outlines characteristics of the found publications. Chapter four presents the identified requirements in detail. The requirements are supported by examples selected from literature. Chapter five includes an evaluation of the resulting requirements on the basis of interviews conducted with industry experts. Chapter six includes this study's limitations and starting points for future research. Lastly, chapter seven draws a conclusion.

\section{Methodology}

A systematic literature review following vom Brocke et al. was conducted to answer the research question [6]. At first, relevant scientific contributions were sought in the databases Google Scholar, IEEE Xplore, Science Direct, Springer Link and Web of Science. Table 1 shows the search string combinations used to find relevant literature. The left column 
includes alternative search strings on anomaly detection and streaming analytics. These are combined with alternative search strings on Industrie 4.0 resp. equivalent terms shown in table 1's right column. The Industrie 4.0 equivalents were found subsequently in the identified literature. I.e., the German term Industrie 4.0 was the first term on table 1's right column, publications resulting from using this term for literature research included the terms Industry 4.0 and intelligent manufacturing. Further literature research on the latter terms resulted in the remaining equivalents. Google trend analysis shows that contributions to the Industrie 4.0 domain gained momentum after 2013 in terms of number of publications, so that the search was restricted to scientific publications after 2013. The search covers publications that included the search string combinations in title, abstract or text.

Table 1: Search string combinations

\begin{tabular}{ccc}
\hline OR & OR \\
\hline Anomaly detection & & Industrie 4.0 \\
Outlier detection & & Industry 4.0 \\
Real-time analytics & A & Intelligent manufacturing \\
Sensor data analysis & $\mathbf{N}$ & Smart factory \\
Stream processing & $\mathbf{D}$ & Smart manufacturing \\
Streaming analytics & & \\
\hline
\end{tabular}

The search initially resulted in 406 publications. Table 2 shows the steps taken to eliminate identified publications that do not fit this paper's research purpose. The findings presented in the following build on the remaining 44 cited publications. The identification of requirements as presented in chapter 4 bases on the recognition of concepts that were explicitly denominated as requirements or frequently reoccur in the cited publications. Specific requirements were identified in a structured approach that consists of three steps. In a first step, the literature was examined for common requirement areas. This examination resulted in the identification of three requirement areas. Secondly, a closer assessment of the requirement areas aimed to identify requirement categories. Lastly, the requirement categories identified in literature were subject to closer assessment to identify specific requirements.

\section{Findings of literature}

Most of the cited publications contribute with a case study, a framework or a combination of both. Other publications contain the development of algorithms or literature reviews, e.g.
The majority of authors do not go into detail on the industries their contribution applies to as shown in Table 3. The most frequently mentioned industries are aerospace and energy with four cited publications each.

Table 2: Elimination steps of structured literature review

\begin{tabular}{|c|c|c|}
\hline $\begin{array}{l}\text { Elimination } \\
\text { measure }\end{array}$ & $\begin{array}{c}\text { No. of } \\
\text { eliminated } \\
\text { publications }\end{array}$ & $\begin{array}{l}\text { Reason for } \\
\text { elimination }\end{array}$ \\
\hline \multicolumn{3}{|c|}{ Screening $(n=406)$} \\
\hline $\begin{array}{l}\text { Screen for } \\
\text { duplicates }\end{array}$ & 15 & Duplicates \\
\hline $\begin{array}{l}\text { Screen for non- } \\
\text { scientific } \\
\text { publications }\end{array}$ & 3 & $\begin{array}{l}\text { Exclusion of } \\
\text { whitepapers }\end{array}$ \\
\hline \multicolumn{3}{|c|}{ Coarse assessment $(\mathrm{n}=388)$} \\
\hline Assess titles & 211 & $\begin{array}{c}\text { Lacking relation } \\
\text { to research } \\
\text { purpose }\end{array}$ \\
\hline $\begin{array}{l}\text { Assess } \\
\text { abstracts }\end{array}$ & 108 & $\begin{array}{l}\text { Lacking or } \\
\text { deviating focus } \\
\text { that does not fit } \\
\text { research purpose }\end{array}$ \\
\hline \multicolumn{3}{|c|}{ Fine assessment $(n=69)$} \\
\hline $\begin{array}{l}\text { Resulting } \\
\text { publications }\end{array}$ & 25 & $\begin{array}{l}\text { Publication } \\
\text { background, focus } \\
\text { or result do not fit } \\
\text { research purpose }\end{array}$ \\
\hline $\begin{array}{l}\text { Resulting } \\
\text { publications }\end{array}$ & 44 & \\
\hline
\end{tabular}

Table 3: Spread of contributions and industries

\begin{tabular}{ccc}
\hline Contribution & Industry & Frequency \\
\hline & Not specified & 4 \\
& Energy & 3 \\
& Aerospace & 2 \\
Case study & Food logistic & 2 \\
& Health & 2 \\
& Transportation & 2 \\
& Optic & 1 \\
& Automotive & 1 \\
\hline \multirow{5}{*}{ Framework } & Not specified & 9 \\
& Automotive & 1 \\
& Bio-medicine & 1 \\
& Energy & 1 \\
& Food & 1 \\
\hline \multirow{7}{*}{ Other } & Not specified & 17 \\
& Aerospace & 2 \\
\hline
\end{tabular}


Table 4 provides further details on the distribution of the kinds of contributions and industries. The cited publications divide into seven groups in terms of purpose. The most frequent purpose refers to algorithms developed to detect anomalies. The improvement of monitoring and control in production environments constitutes the second most frequently pursued goal. Only two publications refer to the development of data preparation techniques to increase data transparency and to enable more standardized data analysis.

\section{Table 4: Purpose of cited publications}

\begin{tabular}{|c|c|}
\hline Purpose & Frequency \\
\hline $\begin{array}{l}\text { Development and testing of algorithms } \\
\text { to detect anomalies in production. }\end{array}$ & 15 \\
\hline $\begin{array}{l}\text { Improvement of production process } \\
\text { and machine monitoring and control on } \\
\text { the basis of real-time data } \\
\text { transparency. }\end{array}$ & 12 \\
\hline $\begin{array}{l}\text { Transformation of } \begin{array}{r}\text { production } \\
\text { environment } \\
\text { towards }\end{array} \\
\text { analytics to monitor machine behavior. }\end{array}$ & 6 \\
\hline $\begin{array}{l}\text { Calculation of anomalies' impacts and } \\
\text { their mitigation. }\end{array}$ & 3 \\
\hline $\begin{array}{l}\text { Review of streaming analytics } \\
\text { frameworks and implementations to } \\
\text { reduce anomalies in production } \\
\text { environments. }\end{array}$ & 3 \\
\hline $\begin{array}{l}\text { Increase of product quality and safety } \\
\text { through the reduction of unexpected } \\
\text { machine behavior. }\end{array}$ & 3 \\
\hline $\begin{array}{l}\text { Data preparation for real-time data } \\
\text { transparency and analysis. }\end{array}$ & 2 \\
\hline
\end{tabular}

\section{Requirements}

To obtain a comprehensive structure, the requirements were divided into the areas of data, infrastructure and analysis, as shown in table 5. These requirement areas subsume ten requirement categories, among which four divide into two or three specific requirements.

\subsection{Data requirements}

The data requirements identified divide into requirements for data source and data composition.

\section{Data source}

Literature agrees on sensors as the most relevant source for data generation in Industrie 4.0. Sensors

Table 5: Requirements overview

\begin{tabular}{|c|c|c|c|}
\hline & $\begin{array}{c}\text { Requirement } \\
\text { categories }\end{array}$ & $\begin{array}{c}\text { Specific } \\
\text { requirements }\end{array}$ & Frequency \\
\hline \multirow{4}{*}{ 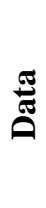 } & Data source & Sensors & 27 \\
\hline & & $\begin{array}{l}\text { Domain } \\
\text { knowledge }\end{array}$ & 8 \\
\hline & & Historical data & 7 \\
\hline & Data composition & & 7 \\
\hline \multirow{7}{*}{ } & Responsiveness & Scalability & 13 \\
\hline & & Latency & 10 \\
\hline & Post-processing & $\begin{array}{l}\text { Reconfigura- } \\
\text { bility }\end{array}$ & 12 \\
\hline & & Evaluation & 8 \\
\hline & & Notification & 5 \\
\hline & Communication & & 13 \\
\hline & Security & & 6 \\
\hline \multirow{5}{*}{ 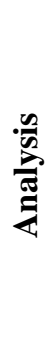 } & Data preparation & & 10 \\
\hline & Data processing $n$ & hode & 5 \\
\hline & $\begin{array}{l}\text { Analysis } \\
\text { methodology }\end{array}$ & $\begin{array}{l}\text { Supervised and } \\
\text { unsupervised }\end{array}$ & 10 \\
\hline & & $\begin{array}{l}\text { Supervised or } \\
\text { unsupervised }\end{array}$ & 5 \\
\hline & $\begin{array}{l}\text { Analysis } \\
\text { supervision }\end{array}$ & & 13 \\
\hline
\end{tabular}

advanced from simple, mechanical devices to smart multi-sensory gadgets that allow real-time data access through digital interfaces. They enable Industrie 4.0 as they interconnect devices by communicating data that would else be unobtainable. Based on these data, production can be adjusted and machine groups can reconfigure. Without sensors, machine controllers would lack overview, which would hinder machine group control and thus autonomous production [7]. Kammerer et al. assess condition parameters of a packaging machine that loads pharmaceutical products into a box. The assessment bases on sensors that measure energy consumption and product location [8]. These parameters constitute the input for classification algorithms determining whether machine behavior deviates from expectation. As the sensors provide a multivariate continuous time-series, machine controllers can use the data for visual monitoring of different interdependent machine conditions.

Other cited publications point out that sensors do not only serve the elicitation of machine data, but also of production environment data [9], [10]. This becomes relevant in case environmental conditions influence production efficiency. Juric et al. prove sensors' 
widespread application potential measuring various factors along a food cold chain, such as room temperature, humidity or light [9]. All sensors are part of a network that enables permanent surveillance of required conditions. Also, the data generated by the network constitutes a basis for process optimization along the food cold chain as inefficiencies become transparent.

Experts' domain knowledge as well as historical data constitute other data sources identified in the cited publications. Different from sensors, domain knowledge and historical data are not used for data generation, but for the enrichment of data analysis. Syafrudin et al. develop a framework, which uses realtime sensor data to predict manufacturing product quality [11]. To this end, data processing includes the offline storage of sensor data and previous analysis results in a database. This historical data is used as input parameters for product quality prediction algorithms. The inclusion of historical data increases prediction accuracy and thus product quality. Li et al.'s framework aims to structure the analysis of data rich, complex processes [12]. The authors propose event pattern matching for the identification of unexpected behavior along manufacturing. The design of event patterns requires judgement of experts, who can define process behavior that is expected in manufacturing. Thus, their anomaly detection approach depends on expert judgement data.

\section{Data composition}

Regarding the composition of data used for realtime analytics, literature shows the requirement to combine timestamps with measurement data. The succession of timestamps enables the creation of timeseries that is necessary for continuous real-time monitoring.

\subsection{Infrastructure requirements}

Infrastructure requirements refer to the technical capabilities required in Industrie 4.0 to implement realtime analysis for anomaly detection. Four categories of infrastructural requirements were identified, namely requirements for technical responsiveness, postprocessing and regarding the setup for data communication as well as infrastructural security.

\section{Responsiveness}

The usefulness of real-time data processing builds on high responsiveness resp. short waiting times [13]. Scalability is required to avoid lagging responses in case of infrastructural extensions or unexpectedly high data volumes. 13 cited publications explicitly mention scalability as a requirement for anomaly detection. Analytical infrastructures have to be extensible, e.g. in case the frequency of sensor data emission rises. The proliferation of data generation and communication devices that comes with new machines or a tighter integration of sensors to production environments is only possible in scalable setups [14]. A frequently used measure to increase scalability for Industrie 4.0 analytics is the parallelization of data processing pipelines [11]. Some authors, such as Zalhana, Silaghi and Buchmann, Peres et al. or Han et al. achieve scalability of sensor generated streaming data by deploying custom software that allows demandoriented addition of servers [15], [16], [17].

Several publications refer to the reduction of latency in different data processing steps, such as data generation, analysis and result evaluation [18]. Measures to keep latency low are increases in computation power as well as standardized communication interfaces. Zalhan, Silaghi and Buchmann put low-latency considerations at the core of their development of a data processing pipeline that serves the real-time generation of insights on sensor data [15]. The authors minimize latency by modularizing data processing and experimenting with the order of steps in their data pipeline [15].

\section{Post-processing}

Identified infrastructural requirements that are relevant after real-time data analysis divide into evaluation, notification and reconfigurability. Evaluation refers to the performance of algorithms or of entire real-time analysis architectures. The reasons for the requirement of post-processing algorithm evaluation are constantly changing input values and altering production systems [19]. Frequent algorithm assessment enables not only the choice of the most fitting one, but also the adjustment of algorithmic parameters.

Five cited publications describe the generation of notifications to alert the need for assessment. In case of unexpected production machine behavior, messenger systems connected to the analysis infrastructure trigger alarms or text messages. Employees, who are affected by the production anomaly receive the notifications and may take corrective action [20], [17].

12 cited publications point out the necessity of system reconfigurability. In case of detected anomalies, the production system needs to adapt in order to run as expected. Rakyta et al. explain that system reconfigurability comes with a trade-off [21]. On the one hand, the authors' implementation shows that reconfiguration can increase desired production 
throughput and improve system functionality by reducing the likelihood of anomaly re-occurrence. On the other hand, reconfiguration requires costly flexibility that needs to be considered in design time. Also, each reconfiguration, regardless of its effectiveness, inherits changeover costs.

\section{Communication}

Data communication refers to transfers of sensor data, analysis results and notifications. Standardized communication interfaces and data transfer protocols are required to lower overall latency and to meet realtime constraints. 13 publications explicitly mention the use of protocols as requirement for their communication infrastructure. Most of the authors consider the Message Queuing Telemetry Transport protocol (MQTT) the de facto standard for the transfer of sensor generated stream data. Berry et al. make use of the protocol to ingest sensor data into a data processing cloud in a standardized and automated process [19]. In fact, the MQTT is used most frequently for sensor data transmission throughout the cited publications.

\section{Security}

Protocols and interfaces build the basis for data security. Six cited publications explicitly refer to the importance of data security in real-time anomaly detection. Carvajal Soto, Tavakolizadeh and Gyulai point out that the implementation of a sensor data analysis system would not be possible without sufficient security measures to maintain correct data accessible only by confidential employees [14]. Security measures identified in the cited publications include trusted devices, data encryption and network protection across all infrastructural components [22], [23], [24].

\subsection{Analysis requirements}

The analysis requirements reflect algorithmic and analytical capabilities necessary to leverage potentials for real-time anomaly detection in Industrie 4.0 environments.

\section{Data preparation}

Ten publications mention data preparation as prerequisite for subsequent value extraction. Preparation of sensor data includes the integration of data with different formats or types as well as cleansing erroneous or irrelevant data. Schutze et " al. indicate that the variety of parameters measured by sensors comes at the cost of data preparation [7]. Lacking data preparation hinders data compatibility and therefore joint analysis. Following Ding et al., missing values, inconsistencies and unexpected values are the main reasons for poor data quality that hinders value extraction [25]. The authors propose a framework with the aim to generate high quality data from sensor streams. However, the requirement to preprocess data leads to a trade-off in the real-time analysis context. Clean data foster better analysis results and more standardized analytical processes, but pre-processing as additional step between data generation and analysis must not take too much time to meet streaming analytics constraints.

\section{Data processing mode}

The majority of the cited publications refers to realtime analysis as online data processing. Data generated by sensors are captured, analyzed and then discarded. This has the advantage that the data does not have to be organized and managed in databases. On the downside, results are less comprehensible after analysis as the processed data is no more available.

Five publications explicitly mention a combination of online and offline analysis. Data analyzed in realtime is stored in a database after processing.

Marz and Warren outline a mass data analysis architecture that modularizes architecture components according to analysis needs [13]. The setup inherits modules for real-time and batch data processing. This so-called Lambda architecture enables real-time views as well as batch views on historicized, integrated data as response to user queries. Building on the Lambda architecture, Berry et al. implement a system that enables real-time processing of sensor data and the analysis of stored historical data [19]. They assess the performance of anomaly detection algorithms offline to evaluate in which cases which algorithms serve best to detect anomalous machine behavior.

\section{Analysis methodology}

The methodologies used to gain actionable insight on real-time data divide into supervised and unsupervised machine learning. Regarding supervised machine learning approaches, cited publications show the use of various data sources for algorithm training. Ali, Patel and Breslin present the case of a company that manufactures biomedical products, such as orthopedic knees [20]. The company makes use of regression-based production forecasting to adjust manufacturing processes and targets. To this end, historical data build the basis to train regression algorithms, which forecast production quantity at 
various stages of the process. Identified examples of applied unsupervised machine learning include error forecasting in manufacturing processes through clustering [26]. Clustering algorithms are used to observe repetitive machine processes. The goal is to classify machine behavior that leads to expected outcomes and to differentiate deviant behavior that results in impaired products.

Five publications show the requirement to combine both unsupervised and supervised machine learning techniques.

\section{Analysis supervision}

13 cited publications present visual monitoring systems for real-time data analysis. The proliferation of sensors in Industrie 4.0 only leads to advanced monitoring and control capability in case the data is structured for a systematic overview [17]. Visual monitoring solutions divide into real image monitoring and graph monitoring in case data is not present in image form. Monitoring images of production processes requires domain experts capable of recognizing unexpected production results from images. French, Benakis and Marin-Reyes implement a prototype for robotic re-manufacturing of jet engine compressor blades used for aircraft vehicles [27]. Remanufacturing includes error-prone welding processes, which require monitoring. To this end, a real-time image feed is implemented that enables experts to inspect the work pieces for errors. Different from that, Zenisek et al. develop a dashboard to monitor data generated by machine sensors in real-time [28]. Different views on various sensor data support experts in the visual identification of unexpected occurrences during production.

\section{Evaluation}

This section provides a brief qualitative evaluation of the results on the basis of interviews with industry experts.

\subsection{Methodology}

Four qualitative interviews with industry experts were conducted following the guidelines of Bryman, Bell and Harley [29]. The goal of the evaluation is twofold. On the one hand, the industry experts are supposed to validate the identified requirements. On the other hand, the interview might lead to further requirements that did not result from the structured literature review

Three interviewed industry experts have at least three years of experience working with machine groups in Industrie 4.0 environments in the automotive sector. The fourth interviewee worked for a gear manufacturer that uses Industrie 4.0 machine groups to produce gears for industrial machines.

Each interview consisted of two phases to achieve a combination of unstructured and semi-structured interviews as proposed by Bryman, Bell and Harley [29]. The first part was an unstructured interview. After a short introduction to the topic, the interviewees were asked for requirements of anomaly detection in machine groups in Industrie 4.0. The unstructured interview allowed for an unrestricted, narrative answer. For the second phase, the interviewees were presented with table 5. After a short explanation, they were asked to comment on the results identified by the structured literature review.

All interviews were conducted separately and took about 45 minutes. The language spoken during the interview was German as all participants are native German speakers. We translated the results afterwards.

\subsection{Results}

The unstructured part of the interviews mostly confirmed the requirements identified through the structured literature review. Table 6 shows the expert interviews' results in relation to the literature review's results in terms of specific requirements resp. requirement categories in case there are no specific requirements. The experts' evaluation splits into the two interview phases in table 6. Two interviews revealed two additional requirements. The experts described the need for production process resp. machine behavior simulation before the first machine run and during production in defined time intervals. These simulations support the definition of expected machine group behavior. When the machines are running, the data generated during production is compared to the simulated data, which enables anomaly identification. Also, the interviews show a requirement for extensive machine testing to determine thresholds for values generated during production. These thresholds can vary among machine groups.

During the semi-structured part, the interviewees confirmed all identified data requirements. Two of them stressed the importance of domain knowledge as basis for anomaly detection. Regarding infrastructural requirements, the interviewees do not conform with internet protocols as method for sensor data communication. According to three of them, the data is mostly transferred through wires to a main computer. The other respondent confirms and adds that wireless sensor data communication is on experimental level. The industry experts could not assert to scalability as requirement due to lacking experience. All 
interviewees emphasized the importance of latency in the real-time context. Two of them added that the entire production machine group stops in case a latency threshold is passed as this means that only lagged data is available. Also, all interviewees agree on the high importance of real-time machine data security. In their experience, the data is stored and processed in an internal infrastructure that does not enable access from outside the factory.

Table 6: Requirements summary

\begin{tabular}{lcc}
\hline & Unstructured & Semi-structured \\
\hline Sensors & $\checkmark$ & $\checkmark$ \\
Domain & $\checkmark$ & $\checkmark$ ! \\
Historical data & $\checkmark$ & $\checkmark$ \\
Data composition & $\checkmark$ & - \\
\hline Scalability & - & $\checkmark$ ! \\
Latency & $\checkmark$ & $\checkmark$ \\
Reconfigurability & $\checkmark$ & $\checkmark$ \\
Evaluation & $\checkmark$ & $\checkmark$ \\
Notification & $\checkmark$ & $\mathbf{x}$ \\
Communication & $\checkmark$ & $\checkmark$ \\
Security & $\checkmark$ & - \\
\hline Data preparation & $\checkmark$ & - \\
Data processing & - & \\
Supervised and & - & \\
unsupervised & & $\checkmark$ \\
Supervised or & - & - \\
unsupervised & & - \\
Analysis & $\checkmark$ & \\
supervision & & \\
Simulation & + & \\
Thresholds & + & \\
\hline \multicolumn{1}{c}{+ added, $\checkmark$ confirmed, } & denied, - none, ! stressed
\end{tabular}

Due to lacking experience, the interviewees could not validate the data requirements of offline and online data processing and analysis methodologies. They confirmed the need of data preparation, which takes place between data generation and visualization for user interfaces. The frequent use of these interfaces that are shown on screens attached to machines or tablets confirms the requirement of analysis supervision, in the interviewees' experience.

\section{Limitations and future research}

The applied methodology as well as the found results have some limitations as presented in the following. Despite the systematic literature review's rigorous approach, there is no guarantee that significant literature contributions were left unconsidered. This may be due to the choice of search strings or the elimination process. Also, the exclusion of contributions from before 2013 might reduce the literature body by relevant publications. Most of the found literature was published as conference contributions, yet only a small share of the literature was published in journals. This may indicate a low level of maturity of the topic. Future researchers, who engage in the same or related fields may increase the topic's rigor and maturity by contributing to journals.

Moreover, some cited publications lack the explicit reference to requirements. Concepts that reoccurred in various publications were considered requirements although the authors do not denominate them as such. As literature on machine groups is also scarce, not all cited publications explicitly mention these, but e.g. refer to dependent production processes on different machines in the same plant [8].

The found requirements are independent from specific industries. However, some of the cited publications indicate that requirements significantly differ across industries. E.g., food cold chain logistics impose different analytical requirements than $\mathrm{CNC}$ machines in the aerospace industry [9], [26]. Requirements regarding the infrastructural setup may also differ with the size of the production plant and the number of the connected devices and data generating sensors.

This paper lays a foundation for further contributions, as to the best of our knowledge, there are no studies on industry neutral requirements for realtime data analysis of production machine group data in Industrie 4.0. The structured and modular presentation of the results in table 5 invites future researchers to extend the requirements by areas, categories or specific requirements. Further, the interviews' unstructured parts provide a basis for research on more requirements.

Follow-up research may take a broader scope that includes entire machine parks and broader real-time data landscapes. A broader scope may also include both batch and real-time processing in its research question, as this paper shows the requirement to combine both approaches. Moreover, future researchers and practitioners may use this paper's findings as a basis for a real implementation, which would verify the findings and may result in the discovery of new requirements.

\section{Conclusion}

Streaming analytics provide promising methodologies to master the vast data proliferation in Industrie 4.0 environments. Real-time data of production machine groups are specifically interesting as the machines work interdependently. Transparency over production data combined with streaming analytics methodologies build the basis for the 
exploration and mitigation of anomalies during production.

A structured literature review was conducted to find requirements for the implementation of real-time anomaly detection mechanisms to improve machine group performance in Industrie 4.0. The search resulted in 44 publications. On this basis, this paper contributes with a structured presentation of these requirements ranging from high-level requirement areas to specific requirements. The synthesis of literature also enables an outlining of examples. The most frequent requirements identified in literature are the use of various sensors as data generators, scalability of data processing infrastructures and standardized communication interfaces that enable rapid data transmission and information generation. Qualitative interviews with industry experts resulted in a validation and extension of these requirements.

This paper contributes to both practice and research. From a researcher's perspective, the identified requirements constitute a starting point for theoretical advancements, such as an extension of table 5. Also, researchers might use this paper as basis for a prototypical implementation that meets the outlined requirements. Practitioners might use the contribution as guideline for the implementation of real-time analytics for anomaly detection in machine groups deployed in Industrie 4.0 environments.

\section{References}

[1] F. Tao, F. Sui, A. Liu, Q. Qi, M. Zhang, B. Song, Z. Guo, S. C.-Y. Lu, and A. Nee, "Digital twindriven product design framework," International Journal of Production Research, vol. 57, no. 12, pp. 3935-3953, 2019.

[2] H. Kagermann, W. Wahlster, and J. Helbig, "Securing the future of german manufacturing industry: Recommendations for implementing the strategic initiative industrie 4.0," Final report of the Industrie, vol. 4, no. 0, 2013.

[3] J. Wang, C. Liu, M. Zhu, P. Guo, and Y. Hu, "Sensor data based system-level anomaly prediction for smart manufacturing," in 2018 IEEE International Congress on Big Data (BigData Congress), pp. 158-165, IEEE, 2018.

[4] R.-J. Hsieh, J. Chou, and C.-H. Ho, "Unsupervised online anomaly detection on multivariate sensing time series data for smart manufacturing," in 2019 IEEE 12th Conference on Service-Oriented Computing and Applications (SOCA), pp. 90-97, IEEE, 2019.

[5] T. Kolajo, O. Daramola, and A. Adebiyi, "Big data stream analysis: a systematic literature review," Journal of Big Data, vol. 6, no. 1, p. 47, 2019.
[6] J. Vom Brocke, A. Simons, K. Riemer, B. Niehaves, R. Plattfaut, and A. Cleven, "Standing on the shoulders of giants: Challenges and recommendations of literature search in information systems research," Communications of the Association for Information Systems, vol. 37, no. 1, p. 9, 2015.

[7] A. Schutze, N. Helwig, and T. Schneider, "Sensors . 4.0-smart sensors and measurement technology enable industry 4.0," Journal of Sensors and Sensor systems, vol. 7, no. 1, pp. 359-371, 2018.

[8] K. Kammerer, B. Hoppenstedt, R. Pryss, S. Stokler, . J. Allgaier, and M. Reichert, "Anomaly detections for manufacturing systems based on sensor data-insights into two challenging realworld production settings," Sensors, vol. 19, no. 24, p. 5370, 2019.

[9] P. Juric, M. B. Bakaric, X. Wang, X. Zhang, and M. Matetic, "Mining data streams for the analysis of parameter fluctuations in iot-aided fruit coldchain.," Annals of DAAAM \& Proceedings, vol. 27, 2016.

[10] Y.-H. Wu, S.-D. Wang, L.-J. Chen, and C.-J. Yu, "Streaming analytics processing in manufacturing performance monitoring and prediction," in 2017 IEEE International Conference on Big Data (Big Data), pp. 3285-3289, IEEE, 2017.

[11] M. Syafrudin, N. L. Fitriyani, D. Li, G. Alfian, J. Rhee, and Y.-S. Kang, "An open source-based real-time data processing architecture framework for manufacturing sustainability," Sustainability, vol. 9, no. 11, p. 2139, 2017.

[12] S. Li, W. Chen, J. Hu, and J. Hu, "Aspie: a framework for active sensing and processing of complex events in the internet of manufacturing things," Sustainability, vol. 10, no. 3, p. 692, 2018.

[13] N. Marz and J. Warren, Big Data: Principles and best practices of scalable realtime data systems. Manning Publications Co., 2015.

[14] J. Carvajal Soto, F. Tavakolizadeh, and D. Gyulai, "An online machine learning framework for early detection of product failures in an industry 4.0 context," International Journal of Computer Integrated Manufacturing, vol. 32, no. 4-5, pp. 452-465, 2019.

[15] P.-G. Zalhana, G. Silaghia, and R. Buchmannna, "Towards a semantic edge processing of sensor data in a smart factory,"

[16] R. S. Peres, A. D. Rocha, P. Leitao, and J. Barata, "Idarts-towards intelligent data analysis and realtime supervision for industry 4.0," Computers in Industry, vol. 101, pp. 138-146, 2018.

[17] S. Han, T. Gong, M. Nixon, E. Rotvold, K.-Y. Lam, and K. Ramamritham, "Rt-dap: A real-time data analytics platform for large-scale industrial 
process monitoring and control," in 2018 IEEE International Conference on Industrial Internet (ICII), pp. 59-68, IEEE, 2018.

[18] S. Trinks, "A classification of real time analytics methods. an outlook for the use within the smart factory," Zeszyty Naukowe. Organizacja i Zarzadzanie/Politechnika Slaska , 2018.

[19] C. Berry, G. Hall, B. Matuszewski, and L. Shark, "A comparison of architectures and evaluation of metrics for in-stream machine learning algorithms in industry 4.0 applications.," 30th International Conference on Condition Monitoring and Diagnostic Engineering Management, pp. 220 226, 2017.

[20] M. I. Ali, P. Patel, and J. G. Breslin, "Middleware for real-time event detection and predictive analytics in smart manufacturing," in 2019 15th International Conference on Distributed Computing in Sensor Systems (DCOSS), pp. 370376, IEEE, 2019.

[21] M. Rakyta, M. Fusko, M. Haluska, and P. Grzn ` ar, 'Maintenance support system for reconfigurable manufacturing systems," in Annals of DAAAM Proceedings of the 26-th DAAAM International symposium on intelligent manufacturing and automation, pp. 1102-1108, 2016.

[22] B. Leang, R.-W. Kim, and K.-H. Yoo, "Real-time transmission of secured plcs sensing data," in 2018 IEEE International Conference on Internet of Things (iThings) and IEEE Green Computing and Communications (GreenCom) and IEEE Cyber, Physical and Social Computing (CPSCom) and IEEE Smart Data (SmartData), pp. 931-932, IEEE, 2018.

[23] C. AgOSTINHO, J. PEREIRA, J.-P. LORRE, S. GHIMIRE, and Y. BENAZZOUZ, "A distributed middleware solution for continuous data collection in manufacturing environments," Enterprise Interoperability in the Digitized and Networked Factory of the Future, pp. 159-166, 2016.

[24] T. Wijaya, W. Caesarendra, B. Pappachan, T. Tjahjowidodo, A. Wee, and M. Roslan, "Robot control and decision making through real-time sensors monitoring and analysis for industry 4.0 implementation on aerospace component manufacturing," in 2017 IEEE Pacific Rim Conference on Communications, Computers and Signal Processing (PACRIM), pp. 1-6, IEEE, 2017.

[25] X. Ding, H. Wang, J. Su, Z. Li, J. Li, and H. Gao, "Cleanits: a data cleaning system for industrial time series," Proceedings of the VLDB Endowment, vol. 12, no. 12, pp. 1786-1789, 2019.
[26] A. Corallo, A. Crespino, C. Dibiccari, M. Lazoi, and M. Lezzi, "Processing big data in streaming for fault prediction: an industrial application," in 2018 14th International Conference on SignalImage Technology \& Internet-Based Systems (SITIS), pp. 730-736, IEEE, 2018.

[27] R. French, M. Benakis, and H. Marin-Reyes, "Intelligent sensing for robotic re-manufacturing in aerospace-an industry 4.0 design-based prototype," in 2017 IEEE International Symposium on Robotics and Intelligent Sensors (IRIS), pp. 272-277, IEEE, 2017.

[28] J. Zenisek, J. Wolfartsberger, C. Sievi, and M. Affenzeller, "Streaming synthetic time series for simulated condition monitoring," IFACPapersOnLine, vol. 51, no. 11, pp. 643-648, 2018.

[29] E. Bell, A. Bryman, and B. Harley, Business research methods. Oxford university press, 2018.

[30] N. Akram, S. Siriwardene, M. Jayasinghe, M. Dayarathna, I. Perera, S. Fernando, S. Perera, U. Bandara, and S. Suhothayan, "Anomaly detection of manufacturing equipment via high performance rdf data stream processing: Grand challenge," in Proceedings of the 11th ACM International Conference on Distributed and Event-based Systems, pp. 280-285, 2017.

[31] A. B. Alaasam, G. Radchenko, and A. Tchernykh, "Stateful stream processing for digital twins: Microservice-based kafka stream dsl," in 2019 International Multi-Conference on Engineering, Computer and Information Sciences (SIBIRCON), pp. 0804-0809, IEEE, 2019.

[32] S. Choi, S. Youm, and Y.-S. Kang, "Development of scalable on-line anomaly detection system for autonomous and adaptive manufacturing processes," Applied Sciences, vol. 9, no. 21, p. 4502, 2019.

[33] H. Huang, S. Ding, L. Zhao, H. Huang, L. Chen, H. Gao, and S. H. Ahmed, "Real-time faultdetection for iiot facilities using gbrbm-based dnn," IEEE Internet of Things Journal, 2019

[34] A. I. Karoly, J. Kuti, and P. Galambos, 'Unsupervised ' real-time classification of cycle stages in collaborative robot applications," in 2018 IEEE 16th World Symposium on Applied Machine Intelligence and Informatics (SAMI), pp. 97-102, IEEE, 2018.

[35] R. Lamberti and L. Stojanovic, "Complex event processing as an approach for real-time analytics in industrial environments," in 2019 IEEE 17th International Conference on Industrial Informatics (INDIN), vol. 1, pp. 220-225, IEEE, 2019.

[36] E. Poormohammady, J. H. Reelfs, M. Stoffers, K. Wehrle, and A. Papageorgiou, "Dynamic algorithm selection for the logic of tasks in iot 
stream processing systems," in 13th International Conference on Network and Service Management (CNSM), pp. 1-5, IEEE, 2017.

[37] J. Queiroz, P. Leitao, and E. Oliveira, "Industrial cyber physical systems supported by distributed advanced data analytics," in International Workshop on Service Orientation in Holonic and Multi-Agent Manufacturing, pp. 47-59, Springer, 2016.

[38] R. Sahal, J. G. Breslin, and M. I. Ali, "Big data and stream processing platforms for industry 4.0 requirements mapping for a predictive maintenance use case," Journal of Manufacturing Systems, vol. 54, pp. 138-151, 2020.

[39] S. Trinks and C. Felden, "Edge computing architecture to support real time analytic applications: a state-of-the-art within the application area of smart factory and industry 4.0," in 2018 IEEE International Conference on Big Data (Big Data), pp. 2930-2939, IEEE, 2018.

[40] S. Trinks and C. Felden, "Image mining for real time fault detection within the smart factory," in 2019 IEEE 21st Conference on Business Informatics $(C B I)$, vol. 1, pp. 584-593, IEEE, 2019.

[41] W. Wang, L. Fan, P. Huang, and H. Li, "A new data processing architecture for multi-scenario applications in aviation manufacturing," IEEE Access, vol. 7, pp. 83637-83650, 2019.

[42] J. Diaz-Rozo, C. Bielza, and P. Larranaga, "Clustering of data streams with dynamic gaussian mixture models: an iot application in industrial processes," IEEE Internet of Things Journal, vol. 5, no. 5, pp. 3533-3547, 2018.

[43] R. Roy, R. Stark, K. Tracht, S. Takata, and M. Mori, "Continuous maintenance and the futurefoundations and technological challenges," Cirp Annals, vol. 65, no. 2, pp. 667-688, 2016.

[44] Y. Cohen, M. Faccio, and A. Elaluf, "Hierarchy of smart awareness in assembly 4.0 systems," IFACPapersOnLine, vol. 52, no. 13, pp. 1508-1512, 2019.

[45] M. Ebrahimi, A. Baboli, and E. Rother, "The evolution of world class manufacturing toward industry 4.0: A case study in the automotive industry," IFAC-PapersOnLine, vol. 52, no. 10, pp. 188-194, 2019.

[46] F. W. Wibowo, "A dynamic intelligent control analysis on the wireless smart machine environments in the industry 4.0," in 2018 International Seminar on Research of Information Technology and Intelligent Systems (ISRITI), pp. 389-393, IEEE, 2018.

[47] T. Munkelt and M. Krockert, "Agent-based selforganization versus central production planning," in 2018 Winter Simulation Conference (WSC), pp. 3241-3251, IEEE, 2018.

[48] A. Elsherif, D. Mandour, M. A. M. Ali, and M. Sedky, "A self-aware paradigm for autonomous architectural systems within the internet of things," International Journal of Parallel, Emergent and Distributed Systems, vol. 32, no. sup1, pp. S15S25, 2017.

[49] S. Bougouffa, K. Meßzmer, S. Cha, E. Trunzer, and B. Vogel-Heuser, "Industry 4.0 interface for dynamic reconfiguration of an open lab size automated production system to allow remote community experiments," in 2017 IEEE International Conference on Industrial Engineering and Engineering Management (IEEM), pp. 2058-2062, IEEE, 2017.

[50] H.-J. Shin, K.-W. Cho, and C.-H. Oh, "Svm-based dynamic reconfiguration cps for manufacturing system in industry 4.0," in Wireless Communications and Mobile Computing, Hindawi, 2018.

[51] W. Lin, Y. Low, Y. Chong, and C. Teo, "Integrated cyber physical simulation modelling environment for manufacturing 4.0," in 2018 IEEE International Conference on Industrial Engineering and Engineering Management (IEEM), pp. 1861-1865, IEEE, 2018.

[52] Y. Zhang, J. Wang, S. Liu, and C. Qian, "Game theory based real-time shop floor scheduling strategy and method for cloud manufacturing," International Journal of Intelligent Systems, vol. 32, no. 4, pp. 437-463, 2017.

[53] A. Khodabakhsh, I. Ar'1, M. Bak'1r, and A. O. Ercan, "Multivariate sensor data analysis for oil refineries and multi-mode identification of system behavior in real-time," IEEE Access, vol. 6, pp. 64389-64405, 2018.

[54] C. M. Ryan, A. Parnell, and C. Mahoney, "Realtime anomaly detection for advanced manufacturing: Improving on twitter's state of the art," arXiv preprint arXiv:1911.05376, 2019. 